apolipoprotein B-100 in NIDDM. Diabetologia. 38:959-967.

15. Sanyal, A.J., et al. 2001. Nonalcoholic steatohepatitis: association of insulin resistance and mitochondrial abnormalities. Gastroenterology.
120:1183-1192.

16. Powell, E.E., et al. 1990. The natural history of nonalcoholic steatohepatitis: a follow-up study of forty-two patients for up to 21 years. Hepatology. 11:74-80.
17. Schattenberg, J.M., Wang, Y., Singh, R., Rigoli, R.M., and Czaja, M.J. 2005. Hepatocyte CYP2E1 overexpression and steatohepatitis lead to impaired hepatic insulin signaling. J. Biol. Chem. 280:9887-9894.

\title{
Caffey disease: an unlikely collagenopathy
}

\author{
Francis H. Glorieux
}

Departments of Surgery, Pediatrics, and Human Genetics, McGill University, and Genetics Unit, Shriners Hospital for Children, Montréal, Quebec, Canada.

Infantile cortical hyperostosis (also known as Caffey disease) is characterized by hyperirritability, acute inflammation of soft tissues, and profound alterations of the shape and structure of the underlying bones, particularly the long bones, mandible, clavicles, or ribs. In this issue of the JCI, Gensure et al. undertook fine mapping of the genetic locus for this disease in a large kindred of individuals with the autosomal dominant form of the condition. The authors found a novel missense mutation in COL1A1, the gene encoding the $\alpha 1$ chain of type I collagen, in all affected individuals in 3 discrete pedigrees (see the related article beginning on page 1250). This is a surprising finding, as all other reported mutations affecting the synthesis of type I collagen lead to conditions such as osteogenesis imperfecta and Ehlers-Danlos syndrome, in which quantitative or qualitative defects in type I collagen synthesis give rise to bone fragility and/or connective tissue hyperextensibility. The deleterious effect of the mutation on collagen fibril morphology is demonstrated; however, the precise functional link between the reported missense mutation and the localized inflammation and hyperostosis seen in Caffey disease awaits future studies.

Infantile cortical hyperostosis (ICH) - also referred to as Caffey or Caffey-Silverman disease - was recognized in 1945 by Caffey and Silverman (1). The condition, later shown to be transmitted as an autosomal dominant trait with incomplete penetrance, becomes clinically evident before 5-7 months of life, and the average age at onset is around 9 weeks. The initial symptom is general hyperirritability, with fever and anorexia, quickly followed by painful, firm soft-tissue swelling, particularly in the face (cheeks and jaws), the scapular region, and upper and lower limbs. It is usually self-limited, with severe symptoms lasting from 2-3 weeks to 2-3 months. The pattern of distribution of the lesions varies from patient to patient and can be symmetrical (Figure 1). Swelling also involves underlying muscle. It is important to

Nonstandard abbreviations used: COL1A1, gene encoding the $\alpha 1$ chain of type I collagen; EDS, EhlersDanlos syndrome; ICH, infantile cortical hyperostosis; PGE, prostaglandin E.

Conflict of interest: The author has declared that no conflict of interest exists.

Citation for this article: J. Clin. Invest. 115:1142-1144 (2005). doi:10.1172/JCI200525148. note that this phase of acute inflammation precedes the abnormal thickening of cortical bone (hyperostosis) that gives its name to the disease. It also subsides long before the hyperostosis resolves. Sometimes lesions recur suddenly in their original sites or in new sites, either during or after the subsidence of the swellings that appeared at the onset of the disease. This uneven protracted clinical course with unpredictable remissions and relapses is one of the most characteristic features of the condition (2).
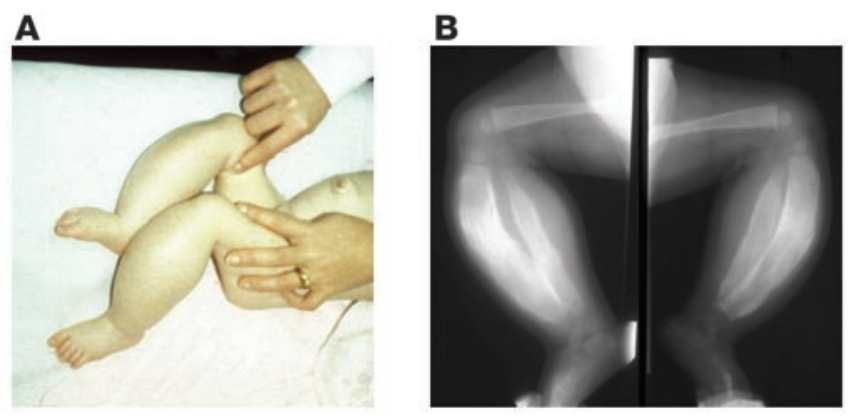

Figure 1

A 6-month-old girl with typical clinical findings of Caffey disease, which appeared at age 7 weeks. The painful swelling of soft tissue around the legs $(\mathbf{A})$ is matched by severe involvement of the tibia and fibula bilaterally (x-radiography, B). Femurs are strikingly unaffected. 
A

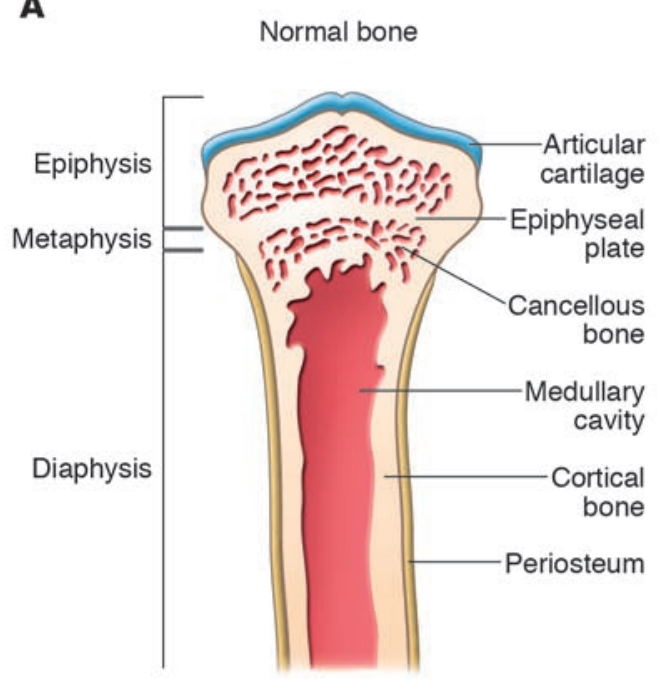

B

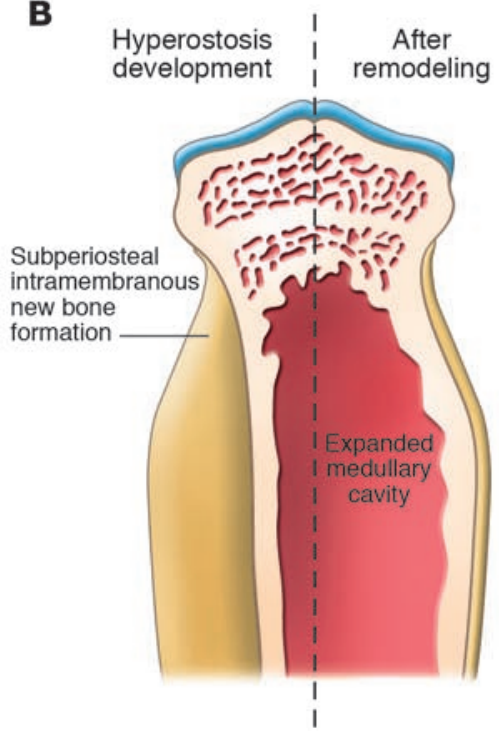

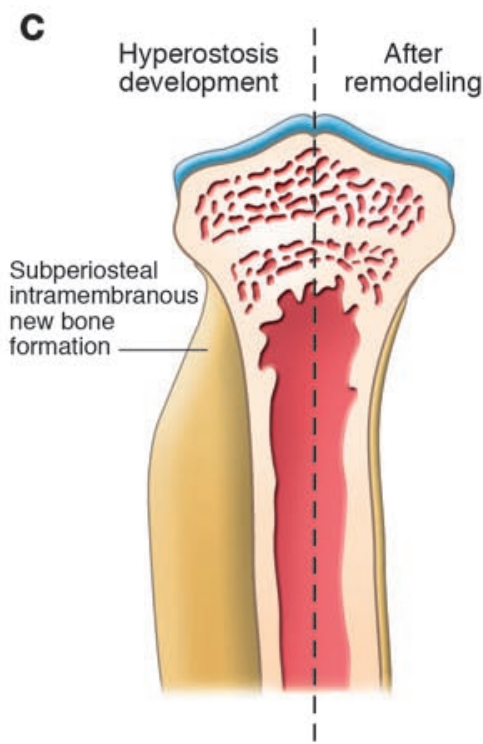

Figure 2

Schematic illustrating normal and exuberant bone formation. (A) Representation of a growing bone. Growth in length is achieved by endochondral bone formation adding cancellous bone in the metaphyseal area. Gain in diameter comes from subperiosteal new bone apposition by intramembranous bone formation. The periosteum is an envelope of fibrous connective tissue that is wrapped around diaphyses. The size of the marrow cavity is controlled by a combination of bone apposition and resorption at the endocortical surface. (B and C) In ICH/Caffey disease, hyperostosis develops by exacerbated subperiosteal intramembranous bone formation triggered by local inflammation (left side of $\mathbf{B}$ and $\mathbf{C}$ ). In the remodeling phase, the excess of bone tissue is resorbed either at the endocortical surface, leading to an expansion of the marrow cavity and a more persistent deformity (right side of B), or at the exocortical surface, with no effect on the size of the marrow cavity (right side of $\mathbf{C}$ ).

local inflammation. Such a hypothesis received strong support from a report by Ueda et al. (4) demonstrating that administration of prostaglandin E (PGE) in infants with ductus-dependent cyanotic heart disease induced a form of cortical hyperostosis that mimics ICH. Two years later, Heyman et al. (5) reported that in 5 affected children, PGE serum levels were significantly elevated and that treatment with indomethacin (an inhibitor of PGE synthesis) rapidly suppressed irritability and led to a progressive decrease in soft-tissue swelling in 6-9 days. Whether elevated PGE levels were a cause or a consequence of the lesions could not be answered. We have made the same observations in 3 patients (Glorieux et al., unpublished data).

\section{ICH pathophysiology}

At a time when the debate regarding how a state of acute bone and soft-tissue inflammation could be limited in time and space and yet be transmitted as an autosomal dominant trait with incomplete penetrance is at a virtual standstill comes the surprising finding reported in this issue of the JCI that ICH is closely associated with a mutation in COL1A1, the gene encoding the $\alpha 1$ chain of type I collagen (6). The careful and elegant work of Gensure et al. has established a strong linkage between the ICH phenotype and the COL1A1 locus on chromosome 17q21 in 3 discrete pedigrees from Australia and Canada with an autosomal dominant form of ICH. More importantly, despite the fact that the families were geographically dispersed and unrelated, it was found that all affected individuals were heterozygous for the identical mutation, a substitution of Arg by Cys at position 836 (R836C), within the helical domain of the $\alpha 1$ chain of type I collagen. The linkage and sequencing data are solid and convincing and represent a major step forward in the understanding of the pathology of an elusive disease.

One has to now try and understand how a point mutation in a widely distributed structural protein can initiate the cascade of inflammatory events that lead to $\mathrm{ICH}$. Type I collagen, the most abundant protein present within bone, is also present in periosteum, ligaments, dermis, and tendon. It is made of a long, continuous triple helix that assembles in highly organized collagen fibrils. The fibrils have high tensile strength, which is critical for the function of bone and other tissues (7). Glycine occurs at every third residue of the triple-helical domain. Because of its small size, it packs tightly at the center of the helix and confers stability. A mutation affecting a Gly residue will thus have an impact on the conformation of the collagen molecule, its integration in the tissue (bone, skin, and tendon), and the structural qualities of the tissue. This is the case in osteogenesis imperfecta, or brittle bone disease, where most mutations within COL1A1 are glycine substitutions. The severity of the phenotype is determined in part by the pattern of inheritance, the position of the mutation in the $\alpha 1$ chain, and the bulkiness of the replacement residue, as it will variably compromise formation and stability of the helix.

In contrast, the R836C mutation identified by Gensure et al. (6) in these ICH patients would not be predicted to have such an effect on the collagen molecule. Why and how it would instead stimulate new bone formation through a process of inflammation has yet to be understood. There has been only one other Arg-to-Cys substitution reported in the $\alpha 1$ chain of type I human collagen. R134C is associated with classical Ehlers-Danlos syndrome (EDS), which is characterized by skin hyperelasticity, joint hypermobility, increased tendency to bruise, and abnor- 
mal scarring (8). Some of the clinical features of EDS are actually evident in the families with ICH studied by Gensure et al. (6), and this makes sense. But the reason why some ICH patients also develop acute bone lesions at a specific age is not clear. The authors hypothesize that periosteum is only loosely attached to the underlying bone structure in infants and that periosteum detachment (perhaps facilitated by the collagen mutation) would be the primum movens leading to increased bone formation. Such a hypothesis is not entirely consistent with the histologic studies done sequentially in cases of ICH (3), which clearly indicate that inflammation is the initial event, in agreement with the clinical and PGErelated observations. It is noteworthy that Gensure et al. found that $79 \%$ of the individuals studied who were heterozygous for the COL1A1 mutation had an episode of cortical hyperostosis, while $21 \%$ of the subjects carrying the R836C substitution do not develop disease, which confirms the clinically observed reduced penetrance at the molecular level. Many unresolved questions regarding the pathology of this disease may have to wait for the engineering of an appropriate mouse model.

In conclusion, by showing convincingly that Caffey disease is associated with a COL1A1 missense mutation, Gensure et al. (6) have once again demonstrated the power of linkage analysis. They have lifted the lid of a black box, but what is inside the box remains to be discovered.

\section{Acknowledgments}

Work in the author's laboratory is supported by the Shriners of North America. The author wishes to thank Frank Rauch for critical reading of the manuscript.

Address correspondence to: Francis H. Glorieux, Genetics Unit, Shriners Hospital for Children, 1529 Cedar Avenue, Montréal, Quebec H3G 1A6, Canada. Phone: (514) 842-5964; Fax: (514) 843-5581; E-mail: glorieux@shriners.mcgill.ca.
1. Caffey, J., and Silverman, W. 1945. Infantile cortical hyperostosis, preliminary report of a new syndrome. American Journal of Rontgenology. 54:1-16.

2. Silverman, F.N. 1985. Infantile cortical hyperostosis. In Caffey's pediatric x-ray diagnosis. F.N. Silverman, editor. Year Book Medical Publishers. Chicago, Illinois, USA. 841-849.

3. Eversole, S.L., Holman, G.H., and Robinson, R.A. 1957. Hitherto undescribed characteristics of the pathology of infantile cortical hyperostosis (Caffey's disease). Bull. John Hopkins Hosp. 101:80-89.

4. Ueda, K., et al. 1980. Cortical hyperostosis following long-term administration of prostaglandin E1 in infants with cyanotic congenital heart disease. J. Pediatr. 97:834-836.

5. Heyman, E., Laver, J., and Beer, S. 1982. Prostaglandin synthetase inhibitor in Caffey disease [letter]. J. Pediatr. 101:314.

6. Gensure, R.C., et al. 2005. A novel COL1A1 mutation in infantile cortical hyperostosis (Caffey disease) expands the spectrum of collagenrelated disorders. J. Clin. Invest. 115:1250-1257. doi:10.1172/JCI200522760.

7. Cole, W.G. 2003. Structure of growth plate and bone matrix. In Pediatric bone, biology and diseases. F.H. Glorieux, J.M. Pettifor, and H. Jüppner, editors. Academic Press. Oxford, United Kingdom/ Boston, Massachusetts, USA. 1-41.

8. Nuytinck, L., et al. 2000. Classical Ehlers-Danlos syndrome caused by a mutation in type I collagen. Am. J. Hum. Genet. 66:1398-1402.

\section{A tax on luxury: HTLV-I infection of $\mathrm{CD} 4{ }^{+} \mathrm{CD} 25^{+}$Tregs}

Robert S. Fujinami

Department of Neurology, University of Utah School of Medicine, Salt Lake City, Utah, USA.

\begin{abstract}
Almost a quarter of a century ago, Oldstone and colleagues proposed that infection of cells by noncytopathic viruses may lead to an alteration of the cells' ability to produce certain products or perform certain tasks, i.e., inhibition of "luxury function." In this issue of the JCI, this topic has been revisited by Yamano et al., who demonstrate that human $T$ cell lymphotropic virus type $\mathrm{I}(\mathrm{HTLV}-\mathrm{I})$ infection of $\mathrm{CD} 4^{+} \mathrm{CD} 25^{+}$Tregs in patients with HTLV-I-associated myelopathy/tropical spastic paraparesis (HAM/ TSP) results in a decrease in FOXP3 mRNA and protein expression (see the related article beginning on page 1361). This leads to the inability of HTLV-I-infected CD4 $4^{+} \mathrm{CD} 25^{+}$Tregs to inhibit the proliferation of $\mathrm{CD}^{+} \mathrm{CD} 25^{-}$Tregs, due to the effect of the HTLV-I tax gene. Defects in the Treg population could be responsible for the large numbers of virusspecific $T$ cells and occurrence of lymphoproliferation and inflammatory autoimmune disease in HAM/TSP patients.
\end{abstract}

Nonstandard abbreviations used: AC, asymptomatic HTLV-I-infected individual; HAM, HTLV-I-associated myelopathy; HD, healthy donor; HTLV-I, human T cell lymphotropic virus type I; TSP, tropical spastic paraparesis.

Conflict of interest: The author has declared that no conflict of interest exists.

Citation for this article: J. Clin. Invest. 115:1144-1146 (2005). doi:10.1172/JCI200525130.
Several years ago, Uchiyama (1) provided interesting insight into the workings of human $\mathrm{T}$ cell lymphotropic virus type I (HTLV-I) infection. He stated that "The interaction between HTLV-I-infected cells with dysregulated function and different kinds of cells in the host, such as lymphocytes and vascular endothelial cells through viral peptides, antigen receptors, cell adhe- sion molecules, and cytokines, appears to be one of the basic mechanisms underlying the development of HTLV-I-associated diseases." In this issue of the JCI, the study by Yamano et al. (2) provides an interesting confirmation of Uchiyama's précis. The authors present data showing that the tax gene of HTLV-I can cause dysfunction of infected $\mathrm{CD} 4{ }^{+} \mathrm{CD} 25^{+}$Tregs and that the interaction between these HTLV-I-infected Tregs and other $\mathrm{CD}^{+}{ }^{+} \mathrm{CD} 25^{-} \mathrm{T}$ cells is dysregulated. One could infer from the study by Oldstone et al. (3) that viral infection of differentiated cells can lead to different outcomes, which depend on what type of cell is infected and what gene product expression is turned off.

HTLV-I is a human retrovirus that is the etiologic agent for HTLV-I-associated myelopathy/tropical spastic paraparesis (HAM/TSP). The prevalence of HAM is about $2.4-3.8 \%$ in HTLV-I-positive individuals $(4,5)$. Therefore, only a minority of infected individuals go on to develop HAM. Yamano et al. (2) explored HTLV-I infection 